\title{
Novel Families of Fractional Quantum Hall States: Pairing of Composite Fermions
}

\author{
John J. Quinn ${ }^{1}$, Arkadiusz Wojs ${ }^{1,2}$, and Kyung-Soo $\mathrm{Yi}^{1,3}$ \\ ${ }^{1}$ University of Tennessee, Knoxville, Tennessee 37996, USA \\ ${ }^{2}$ Wroclaw University of Technology, 50-370 Wroclaw, Poland \\ ${ }^{3}$ Pusan National University, Pusan 609-735, Korea
}

\begin{abstract}
Fractional quantum Hall $(\mathrm{FQH})$ states have recently been observed at unexpected values of the filling factor $\nu$. Here we interpret these states as a novel family of FQH states involving pairing correlations rather than Laughlin correlations among the quasiparticles (QP's). The correlations depend upon the behavior of the $\mathrm{QP}-\mathrm{QP}$ pseudopotential $V_{\mathrm{QP}}\left(L^{\prime}\right)$, the interaction energy of a pair as a function of the pair angular momentum $L^{\prime}$. This behavior, known from numerical studies of small systems, is used to demonstrate that pairing correlations give rise to FQH states at the experimentally observed values of $\nu$.

PACS numbers: 71.10.Pm, 73.43.-f

Keywords: fractional quantum Hall states, pairing of composite Fermions, quasiparticles, pseudopotentials
\end{abstract}

Recently Pan et al. [1] observed fractional quantum Hall minima in $\rho_{x x}$ at unexpected values of the Landau level filling factor $\nu$ outside the Jain sequence of states with $\nu=n(2 p n \pm 1)^{-1}$, where $n$ and $p$ are positive integers. The composite Fermion hierarchy [2, 3] , in which the reapplication of the Chern-Simons (CS) mean field approximation attaches additional flux quanta to the quasiparticles (QP's) in a partially filled composite Fermion $(\mathrm{CF})$ shell, has been suggested [1, 4] as a possible explanation of some of these states. However, the form of the residual interactions [5, 6, 7, 8] between QP's sometimes precludes Laughlin correlations and the realization of certain daughter states of the CF hierarchy [9, 10]. Among these states are the $\nu=4 / 11$ and 4/13 daughter states observed experimentally [1], which correspond to quasielectron (QE) and quasihole (QH) filling factors $\nu_{\mathrm{QE}}=1 / 3$ and $\nu_{\mathrm{QH}}=1 / 5$, respectively. In addition, the observed even denominator fractional fillings cannot arise within the CF hierarchy. Here, we show that instead of having Laughlin correlations, the QP's form pairs, and that these pair excitations cause the novel incompressible daughter states at the unexpected values of $\nu$ observed experimentally.

By Laughlin correlations 11 among interacting Fermions confined to a spherical surface we mean that pair states with the largest values of the pair angular momentum $L^{\prime}$ (or smallest values of the relative angular momentum $\mathcal{R}=2 l-L^{\prime}$, where $l$ is the single Fermion angular momentum) are maximally avoided. Laughlin correlations occur if and only if the pseudopotential $V\left(L^{\prime}\right)$ is "superharmonic," that is, rises with increasing $L^{\prime}$ faster than $L^{\prime}\left(L^{\prime}+1\right)$ as the avoided value of $L^{\prime}$ is approached [6, 7, 9,12 . Here $V\left(L^{\prime}\right)$ is the interaction energy of a pair of Fermions as a function of $L^{\prime}$. The pseudopotential for electrons in the lowest Landau level $(n=0)$ is superharmonic at all values of $L^{\prime}$. However, for the first excited Landau level $(n=1)$ it is not superharmonic at the largest value of $L^{\prime}$, namely $L^{\prime}=2 l-1$. This is known to result in pairing of the electrons [12, 13] at filling factors $\nu=7 / 3,5 / 2$, and $8 / 3$.

For QP's of the Laughlin $\nu=1 / 3$ state, $V_{\mathrm{QP}}\left(L^{\prime}\right)$ has been obtained from exact numerical diagonalization studies of small systems [5, 6, 7, 8, 10] (with particle number $N \leq 12$ ). This is illustrated for QE's in Fig. 17. When plotted as a function of $N^{-1}, V_{\mathrm{QE}}(\mathcal{R})$ converges to a rather well defined limit as shown in Fig. 10 for $\mathcal{R}=1$, 3 , and 5 . The results are quite accurate up to an overall constant (which does not affect the correlations). Because the short-range interactions (i.e., at small values of $\mathcal{R}$ or small QE separation) determine the nature of the ground state, numerical results for small systems describe the essential correlations quite well for systems of any size. Furthermore, the subharmonic character of $V_{\mathrm{QP}}$ at certain values of $\mathcal{R}(\mathcal{R}=1$ for QE's and $\mathcal{R}=3$ for QH's) makes it impossible for $\nu_{\mathrm{QE}}=1 / 3$ and $\nu_{\mathrm{QH}}=1 / 5$ to lead to incompressible daughter states of the $\mathrm{CF}$ hierarchy. By this we mean that for a spin polarized state in which QP's of the Laughlin $\nu=1 / 3$ state yield filling factors $\nu_{\mathrm{QE}}=1 / 3\left(\right.$ or $\left.\nu_{\mathrm{QH}}=1 / 5\right)$, Laughlin correlations among the QP's, giving rise to incompressible daughter states at $\nu=4 / 11$ ( or $\nu=4 / 13$ ), cannot occur [6, 7, 9, 10].

How then can we understand the novel states observed at $\nu=5 / 13,3 / 8,4 / 11$, etc.? To illustrate, we use the case of QE's (the application to QH's is straightforward and will be given elsewhere). It is apparent that $V_{\mathrm{QE}}(\mathcal{R})$ is not superharmonic at $\mathcal{R}=1$. In fact, its maximum repulsion occurs at $\mathcal{R}=3$. In this case, the QE's do not display Laughlin correlations by avoiding the pair state with $\mathcal{R}=1$ (or, in the planar geometry, by having the Laughlin-Jastrow factor $\prod_{i<j}\left(z_{i}-z_{j}\right)^{2}$ in the ground state wavefunction where $z_{j}=x_{j}-i y_{j}$ is the position of the $j^{\text {th }} \mathrm{QE}$ ). Instead, they tend to form pairs with $\mathcal{R}=1$ in order to minimize the pair amplitude 12, 13. with $\mathcal{R}=3$. Pairing at filling factor $\nu=5 / 2$ has been considered by others 14,15$]$ based on the observation of 


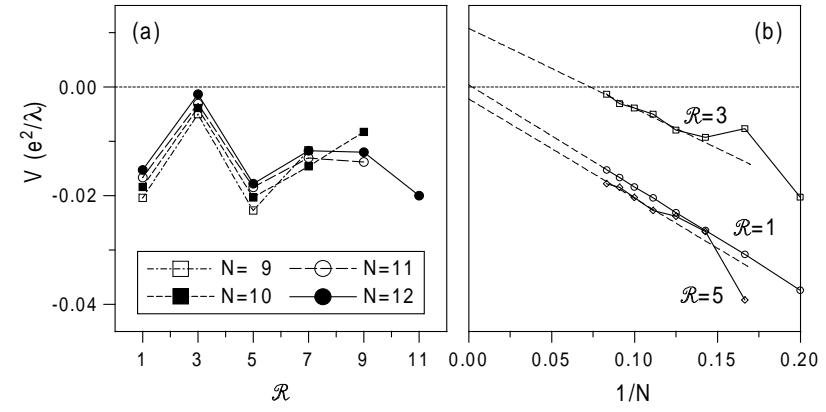

FIG. 1: (a) Interaction pseudopotentials $V_{\mathrm{QE}}(\mathcal{R})$ for a pair of QE's of the Laughlin $\nu=1 / 3$ state, calculated for up to $N=12$ electrons on a spherical surface. (b) The leading QE pseudopotential parameters $V_{\mathrm{QE}}(\mathcal{R})$ for $\mathcal{R}=1,3$, and 5, plotted as a function of $N^{-1}$, inverse of the particle number. Extrapolation to $N^{-1} \rightarrow 0$ corresponds to an infinite planar system.

an unexpected incompressible ground state.

The pairs can be thought of either as Bosons or Fermions with pair angular momentum $l_{\mathrm{P}}=2 l-1$, since in two-dimensional systems Bosons (Fermions) can be transformed into Fermions (Bosons) by a CS transformation [16, 17]. For a system containing more than a single pair, the allowed values of the total angular momentum of two pairs must be chosen in such a way that the Pauli principle is not violated when accounting for identical constituent Fermions belonging to different pairs. This can be accomplished if different pairs are not allowed to approach too closely by requiring that the largest allowed value of the total angular momentum of two pairs (treated as Fermions) to be given by $\tilde{L}^{\prime}=2 l_{\mathrm{FP}}$, where

$$
2 l_{\mathrm{FP}}=2(2 l-1)-\gamma_{\mathrm{F}}\left(N_{\mathrm{P}}-1\right) .
$$

Here the simplest assumption of complete pairing is made, so that $N_{\mathrm{P}}=N / 2$ is the number of Fermion pairs (FP's), and $\gamma_{\mathrm{F}}$ will be an odd integer (if the pairs were treated as Bosons $\gamma_{\mathrm{B}}$ would equal $\left.\gamma_{\mathrm{F}}-1\right) . \quad \gamma_{\mathrm{F}}$ is chosen so that the Fermion pair filling factor $\nu_{\mathrm{FP}}=$ $\left(N_{\mathrm{P}}-1\right) / 2 l_{\mathrm{FP}}$ is equal to unity when the QE filling factor $\nu_{\mathrm{QE}}=(N-1) / 2 l$ is also equal to unity. This condition gives $l_{\mathrm{FP}}=2 l-1-\frac{3}{2}\left(N_{\mathrm{P}}-1\right)$ for the "effective" angular momentum of one FP and

$$
\nu_{\mathrm{FP}}^{-1}=4 \nu_{\mathrm{QE}}^{-1}-3
$$

for large systems. This CS transformation automatically forbids states of two FP's with the smallest separation. The larger pair-pair separation causes the constituent QE's to avoid the largest repulsion at $\mathcal{R}=3$. In addition, the transformation selects from $\mathcal{D}_{L}(N, l)$, the number of multiplets of total angular momentum $L$ formed from $N$ Fermions each with angular momentum $l$, a subset $\mathcal{D}_{L}\left(N_{\mathrm{P}}, l_{\mathrm{FP}}\right)$.

We expect pair formation for QE filling factor satisfying $2 / 3 \geq \nu_{\mathrm{QE}} \geq 1 / 3$, where Laughlin-Jain states

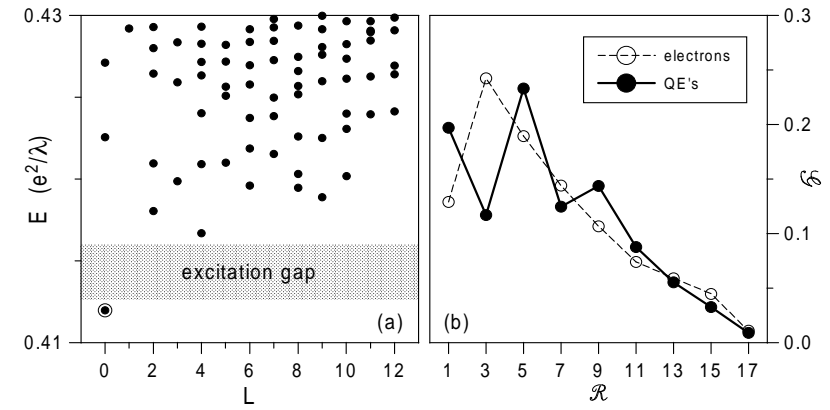

FIG. 2: (a) Low energy spectrum (energy $E$ as a function of total angular momentum $L$ ) of 10 QE's at $2 l=17$ corresponding to $\nu_{\mathrm{QE}}=1 / 2$ and $\nu=3 / 8$, obtained in exact diagonalization in terms of individual QE's interacting through the pseudopotential shown in Fig. 1] $\lambda$ is the magnetic length. The open circle at $L=0$ denotes a robust ground state. (b) Coefficient $\mathcal{G}(\mathcal{R})$, the amplitude associated with pair states of relative pair angular momentum $\mathcal{R}$, for the lowest $L=0$ state of 10 particles in a shell of angular momentum $l=17 / 2$. The solid dots and open circles are obtained for the QE's (the $\nu=1 / 2$ ground state marked in frame a) and electrons, respectively.

[1, 18] that avoid $\mathcal{R}=1$ would normally occur for a superharmonic potential. If we assume the FP's support Laughlin correlations, when their separations are large, then incompressible ground states would be expected at the Laughlin filling factors $\nu_{\mathrm{FP}}=1 / 5,1 / 7$, and $1 / 9$. From Eq.(2), these correspond to values of $\nu_{\mathrm{QE}}$ given by $1 / 2,2 / 5$, and $1 / 3$. For $\nu_{\mathrm{QE}} \geq 1 / 2$ we make use of $\mathrm{QE}-$ QH symmetry and think of Fermion pairs of QH's giving $\nu_{\mathrm{QH}}=1 / 2,2 / 5,1 / 3$ and $\nu_{\mathrm{QE}}=1 / 2,3 / 5$, and $2 / 3$. Only at these values of $\nu_{\mathrm{FP}}$ do we have Laughlin states of the Fermion pairs with $\nu_{\mathrm{QE}}$ in the required range. In the hierarchy scheme 2] describing partially filled CF levels, the original electron filling factor is given by

$$
\nu^{-1}=2+\left(1+\nu_{\mathrm{QE}}\right)^{-1} \text {. }
$$

Here the 2 on the right hand side comes from the addition of two flux quanta per electron in the original CS transformation on the electrons, and $\left(1+\nu_{\mathrm{QE}}\right)$ is the $\mathrm{CF}$ filling factor. Hence, we find new incompressible states at $\nu=5 / 13,8 / 21,3 / 8,7 / 19$, and $4 / 11$. All except the $7 / 19$ and $8 / 21$ states have been observed. We are uncertain whether there is some reason why these two states do not occur, or if, because they have such large denominators, they are simply difficult to see and might be observed in future experiments. It could be that our simple model, which assumes complete pairing of all QP's, is not valid at every value of $\nu_{\mathrm{QP}}$ (alternatively, partially paired states could be considered), or that a spin unpolarized state preempts pair formation.

As an illustration we have performed an exact diagonalization on a system containing $N=10$ QE's at $l=17 / 2$. This corresponds to $\nu_{\mathrm{QE}}=1 / 2$. The energy spectrum (Fig. 2a) obtained using the QE-QE 
pseudopotential given in Fig. 1 shows a robust $L=0$ ground state and an excitation gap. The coefficient $\mathcal{G}(\mathcal{R})=\sum_{L^{\prime} \alpha^{\prime}}\left|G_{L^{\prime} \alpha^{\prime}}(\mathcal{R})\right|^{2}$ appropriate for the $L=0$ ground state is shown in Fig. 20 bor the allowed values of $\mathcal{R}$. $G_{L^{\prime} \alpha^{\prime}}(\mathcal{R})$ is the coefficient of fractional grandparentage [5, 6, 7, 19], and $\mathcal{G}(\mathcal{R})$ is a measure of the amplitude associated with pair states of relative pair angular momentum $\mathcal{R}$. For the purpose of comparison, the same results are shown for the $L=0$ state of a ten-electron system in the lowest Landau level, also at $l=17 / 2$. The large decrease in $\mathcal{G}(\mathcal{R})$ at $\mathcal{R}=3$ and increase at $\mathcal{R}=1$ in going from $V_{e}(\mathcal{R})$ to $V_{\mathrm{QE}}(\mathcal{R})$ is clear evidence of the avoidance of pair states with $\mathcal{R}=3$ and the formation of pairs with $\mathcal{R}=1$ in the case of QE's.

Rather than contradicting the assertion 10 that the $4 / 11$ state (or $\nu=4 / 13$ state when $\nu_{\mathrm{QH}}=1 / 5$ ) cannot occur as an incompressible daughter state in the standard CF hierarchy of Laughlin correlated QP states of a spin polarized system, the results of Pan et al. 1] offer support for the idea of pairing of QP's at certain values of $\nu_{\mathrm{QP}}$. In contrast to the hierarchies of Laughlin correlated QP states, the pairing picture can account for incompressible states in the lowest Landau level with even denominator fractional filling. We emphasize that simple repetition of Laughlin correlations among daughter states 2, 4, 20] containing CF QP's is not always appropriate 10] because of the form of $V_{\mathrm{QP}}(\mathcal{R})$. The proposed pairing of CF QP's gives rise to a new type of QP which in turn leads to a myriad of completely novel hierarchy states.

The authors gratefully acknowledge the support by Grant DE-FG 02-97ER45657 of the Material Research Program of Basic Energy Sciences-US Department of Energy. AW acknowledges support from Grant 2P03B02424 of the Polish KBN, and KSY acknowledges partial support of the ABRL(R14-2002-029-01002-0) through the KOSEF.
[1] W. Pan, H.L. Störmer, D.C. Tsui, L.N. Pfeiffer, K.W. Baldwin, and K.W. West, Phys. Rev. Lett. 90 (2003) 016801.

[2] P. Sitko, K.-S. Yi, and J.J. Quinn, Phys. Rev. B 56 (1997) 12417.

[3] F.D.M. Haldane, Phys. Rev. Lett. 51 (1983) 605; This paper was the first hierarchy scheme, but the quasiparticles were treated as Bosons and their interaction was unexplored.

[4] J.H. Smet, Nature 422 (2003) 391.

[5] P. Sitko, S.N. Yi, K.-S. Yi, and J.J. Quinn, Phys. Rev. Lett. 76 (1996) 3396.

[6] A. Wojs and J.J. Quinn, Philos. Mag. B 80 (2000) 1405.

[7] J.J. Quinn and A. Wojs, J. Phys.: Cond. Matter 12 (2000) R256.

[8] S.-Y. Lee, V.W. Scarola, and J.K. Jain, Phys. Rev. Lett. 87 (2001) 256803.

[9] J.J. Quinn and A. Wojs, Physica E 6 (2000) 1.

[10] A. Wojs and J.J. Quinn, Phys. Rev. B 61 (2000) 2846.

[11] R.B. Laughlin, Phys. Rev. Lett. 50 (1983) 1395.

[12] A. Wojs, Phys. Rev. B 63 (2001) 125312.

[13] A. Wojs and J.J. Quinn, Physica E 12 (2002) 63.

[14] G. Moore and N. Read, Nucl. Phys. B 360 (1991) 362.

[15] M. Greiter, X.G. Wen, and F. Wilczek, Nucl. Phys. B 374 (1992) 567.

[16] J.J. Quinn, A. Wojs, Jennifer J. Quinn, and A.T. Benjamin, Physica E 9 (2001) 701.

[17] See, for example, F. Wilczek, Fractional Statistics and Anyon Superconductivity (World Scientific, Singapore, 1990).

[18] J.K. Jain, Phys. Rev. Lett. 63 (1989) 199.

[19] See, for example, A. de Shalit and I. Talmi, Nuclear Shell Theory (Academic Press, New York, 1963).

[20] R.G. Mani and K. von Klitzing, Z. Phys. B 100 (2000) 635. 